\title{
The role of 18FDG PET/CT imaging of aortic atherosclerosis: prospective study and technique optimization
}

\author{
Amr A. Elfattah Hassan Gadalla ${ }^{*^{*}}$ (D) and Nahla Dessoki Elsayed ${ }^{2}$
}

\begin{abstract}
Background: Atherosclerosis is an inflammatory disease of the inner wall of large and medium-sized arteries. The progress of atherosclerosis based on a lot of factors, including systemic involvement of disease, the precise vascular arterial affection, and the degree of flow obstruction. We aim in this study to estimate the FDG uptake of the aortic wall in the early and delayed imaging and to correlate this with the morphologic changes detected by $\mathrm{CT}$.

Results: This is a prospective study that was performed through 1 year. The study included 50 patients [30 males (60\%) and 20 females (40\%)] with male to female mean ratio 1.5:1 and their mean age 58.3 \pm 15.7 years. Each patient underwent dual time-point 18F-FDG PET CT imaging at $\sim 60$ min (Early images) and 180 min (delayed images) after the administration of 18F-FDG. For each patient, the global 18F-FDG uptake in the aorta was determined by manually drawing regions of interest (ROls) around the outer part of the arterial wall on every slice of the attenuation-corrected transverse PET CT images. Per-patient, per-time-point, pervessel, and per-ROI radiotracer decay-corrected and body weight-corrected SUVS were calculated, resulting in a single mean value of maximum SUV for the aorta. The aortic wall FDG uptake was measured in both early and delayed images and expressed in terms of SUVmax. Then Retention index percentage of the aorta was measured. The retention index percentage was calculated by subtracting the SUVmax in early images from the SUVmax in delayed images and dividing by SUVmax in early images.

Conclusion: Aortic wall FDG uptake can be used as an additional parameter as well as a biomarker on evaluation of the arterial atherosclerotic activity. Delayed post $3 \mathrm{~h} \mathrm{FDG} \mathrm{imaging} \mathrm{is} \mathrm{more} \mathrm{accurate} \mathrm{than} \mathrm{the}$ routine early post $1 \mathrm{~h}$ imaging in evaluating the atherosclerotic activity.
\end{abstract}

Keywords: PET/CT, Aorta, Atherosclerosis, Activity, Dual point

\section{Background}

Atherosclerosis is an inflammatory disease of the inner wall of large and medium-sized arteries [1]. The progress of atherosclerosis is based on a lot of factors, including systemic involvement of disease, the precise vascular arterial affection, and the degree of flow obstruction. Wide range exists, and even many patients with critical obstruction of flow to vital organs may

* Correspondence: dr_amr722@hotmail.com

'Radiodiagnosis, Faculty of Medicine, Cairo University, Giza, Egypt

Full list of author information is available at the end of the article survive for many years, despite the severity of the disease [2]. Atherosclerosis imaging represents a wide range of established and under trial radiological modalities that can be used to detect anatomical and physiological results of long-standing atherosclerosis [3]. Nuclear medicine imaging, such as positron emission tomography (PET), differs from other imaging modalities, since it has the ability to noninvasively detect molecular and cellular processes to identify early disease progress before anatomical manifestations. Moreover, multimodality imaging like PET/CT is very effective, since the computed tomography (CT) can precisely localize the PET findings. PET/CT can be used to study metabolic and physiologic processes 
in vivo with precise anatomic localization. 18FFluorodeoxyglucose (FDG) is the most utilized radiopharmaceutical. Also PET/CT allows quantitative measurements that provide more accurate and less observer-dependent measure of inflammatory atherosclerosis than qualitative assessment [4]. It has become clear that the cellular and extracellular composition of plaques is a primary determinant of plaque stability. The main characteristics include the state of thin fibrous cap, large lipid core, a paucity of smooth muscle cells, and an abundance of inflammatory cells [5]. High-risk plaques by MRI (large lipid core, intra-plaque hemorrhage, or on ultrasound) have greater FDG uptake than those of stable phenotypes. In PET/CT images, FDG uptake is strongly correlated with hyper enhancement of plaque after administration of a iodinated contrast agent and is negatively correlated with the degree of arterial calcification. Finally, arterial FDG uptake is greater in arteries with high pulsed wave velocities, another entity marker of vascular dysfunction [6].

We aim in this study to estimate the FDG uptake of the aortic wall in the early and delayed imaging and to the correlate this with the morphologic changes detected by CT.

\section{Methods}

This prospective study was performed through 1 year. It included 50 patients, 30 males $(60 \%)$ and $20 \mathrm{fe-}$ males (40\%), with mean age $58.3 \pm 15.7$ years. All the patients underwent double time-point 18F-FDG PET/CT with informed consent before enrollment. 18F-FDG PET CT imaging was performed using a dedicated whole-body PET/CT scanner (Philips Ingenuity PET/CT system).

\section{Patients' selection}

The selection process included identifying those patients who fulfilled the criteria given below and approved by the ethical committee.

\section{Inclusion criteria}

- Patients with variable risk categories to have aortic atherosclerosis referred for FDG PET/CT study for different indications.

\section{Exclusion criteria}

- Patients who underwent aortic angioplasty or stenting.

- Patient who underwent surgery including major vascular intervention.
- Patients with recently diagnosed malignant tumors known to be associated with vascular invasion or receiving prior chemotherapy or radiotherapy.

- Patient who were on atherosclerotic therapy.

\section{Medical data}

- Personal history: gender, age, smoking, height, weight and body mass index. History of diabetes mellitus, hypertension, or administration of antiatherosclerotic medication should be documented.

- Present illness:

- Primary indication for PET.

- Stage of primary neoplasm.

- Previous investigation related to aorta.

- Previous treatment: e.g., drugs for dyslipidemia.

- General examination:

- Weight, height, body mass index, pulse rate, blood pressure, and random blood sugar.

\section{Image protocol}

PET images were acquired from mid-skull to mid-thigh in 3D mode and reconstructed in transverse, coronal, and sagittal planes using a 64-row multidetector hybrid system (Philips-Ingenuity PET/CT system) and (GE Healthcare 16-slice system), with an axial field of view (FOV) of $216 \mathrm{~mm}$ and a PET sensitivity of 7.6 counts per second $/ \mathrm{kBq}$.

\section{Patient preparation}

Patients are instructed fasting for at least $6 \mathrm{~h}$ and were asked to avoid strenuous exercise for previous $24 \mathrm{~h}$. Blood glucose was checked in all patients prior to FDG injection. Serum blood glucose levels were determined by finger stick measurement before administration of the radiotracer to ensure a serum glucose level below $200 \mathrm{mg} / \mathrm{dl}$.

All patients were kept in warm and calm environment then received $\sim 5.2 \mathrm{MBq}$ of ${ }^{18} \mathrm{~F}$-FDG per kilogram of body weight intravenously. Each patient underwent multiple time-point ${ }^{18} \mathrm{~F}$-FDG PET CT imaging at $\sim 60$ and $180 \mathrm{~min}$ after the administration of ${ }^{18} \mathrm{~F}$-FDG. At $60 \mathrm{~min}$, the acquisition time per bed position was $2 \mathrm{~min}$. To compensate for radioactive decay of the tracer, the acquisition time per bed position was extended to $4 \mathrm{~min}$ for the 3 -h scan. A low-dose CT-scan for attenuation correction and anatomical orientation as well as to detect aortic calcification was performed at a speed of $0.42 \mathrm{~s}$ per rotation and a section thickness of $5.0 \mathrm{~mm}$. The window level in non-enhanced images was zero Hounsfield Units (HU) settled in water. For 
detection of aortic intimal changes, a bolus intravenous injection of contrast was given to differentiate the aortic wall from the intra-luminal blood. An adequate concentration of contrast medium was obtained at a flow rate of $0.4 \mathrm{ml} / \mathrm{s}$ using a power injector. Enhanced images from the aortic arch to the bifurcation were obtained by injecting a total volume of $50-60 \mathrm{ml}$ of contrast medium.

\section{Image analysis}

The obtained data were interpreted and analyzed both quantitatively and qualitatively as will be mentioned. All images were viewed on a workstation, which showed multiplanar and 3D reconstructions of the PET and CT images as well as quantitative and semi-quantitative measurements. The entire aorta, starting at the ascending aorta and ending at the aortic bifurcation, was assessed. Blinded for time points and patient characteristics, each PET scan was reviewed and quantitatively assessed for the degree of global arterial 18 F-FDG uptake (Figs. 1, 2, and 3) as follows.

\section{PET findings}

For each patient, for each time point, the global ${ }^{18} \mathrm{~F}$ FDG uptake in the aorta was determined by manually drawing regions of interest (ROIs) around the outer part of the arterial wall on every slice of the attenuation-corrected transverse PET CT images. The blood pool ${ }^{18}$ F-FDG activity was determined in the inferior vena cava lumen as a representative of blood pool activity. Per-patient, per-time-point, pervessel, and per-ROI radiotracer decay-corrected and body weight-corrected standard uptake values (SUVs) were calculated, resulting in a single mean value of maximum SUV for the aorta and inferior vena cava. The blood pool FDG activity as well as the aortic wall FDG uptake were measured in both early and delayed images and expressed on terms of SUVmax. Then retention index percentage of the blood pool as well as of the aorta was measured. The retention

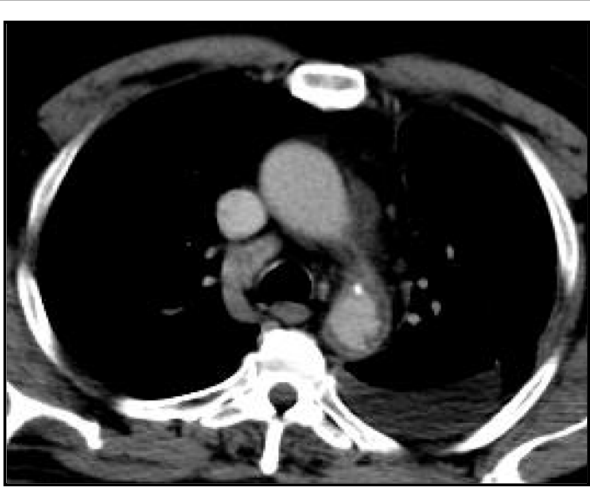

(A)

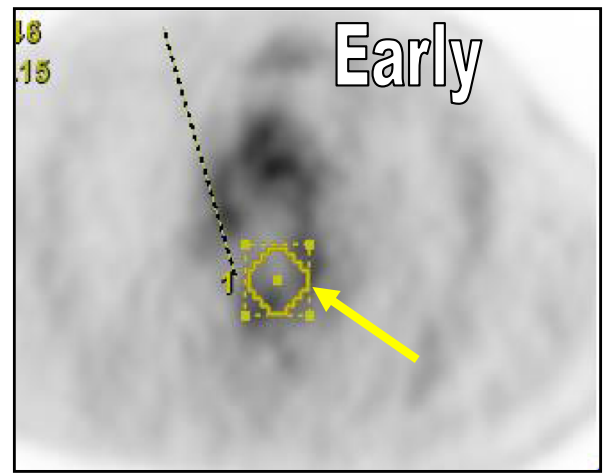

(C)

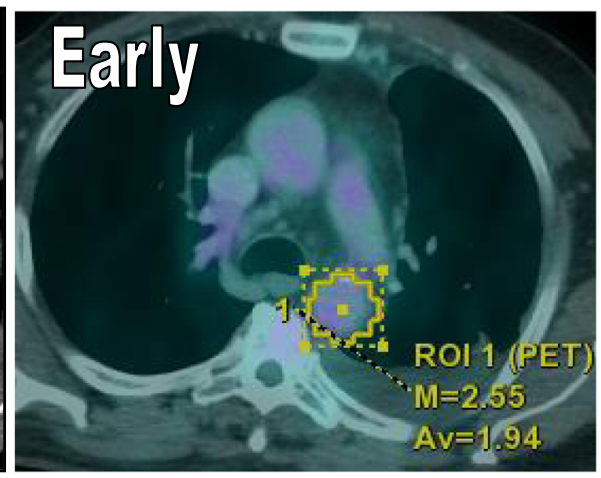

(B)

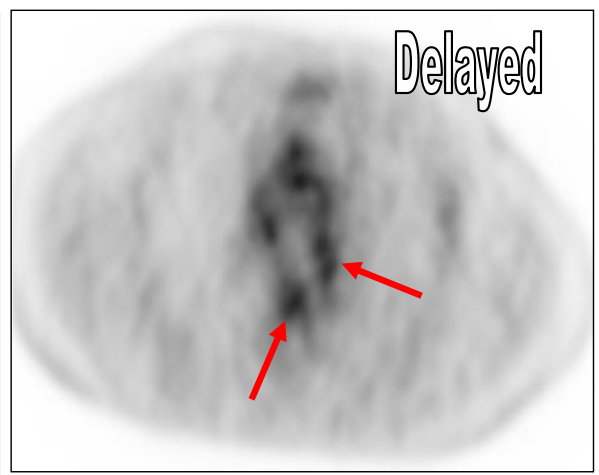

(D)

Fig. 1 Male patient 57-year-old, non-smoker, non-diabetic, non-hypertensive. He had a history of biliary obstruction with CBD stent underwent PET/CT for suspicious of abdominal malignancy. a Post contrast CT images showed diffuse irregular intimal thickening and small calcification (b, c) fused and PET early images showed measurement of SUVmax at the outer wall of the descending thoracic aorta (yellow arrow). $\mathbf{d}$ Delayed PET images showed increased FDG uptake with higher SUVmax (red arrows) 


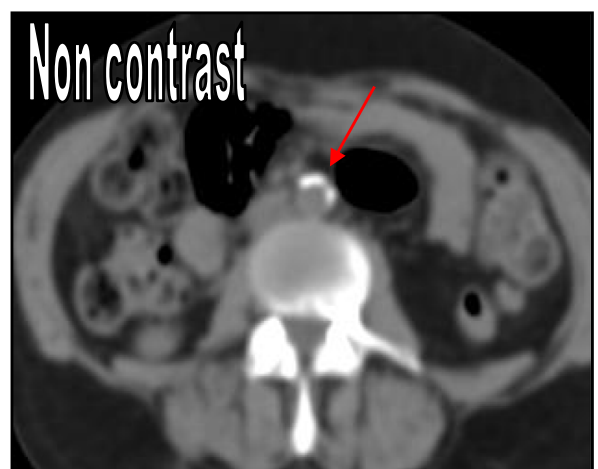

((A)

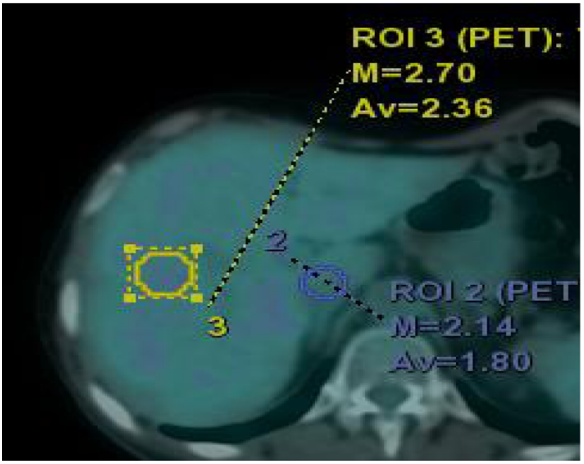

(ㄷ)

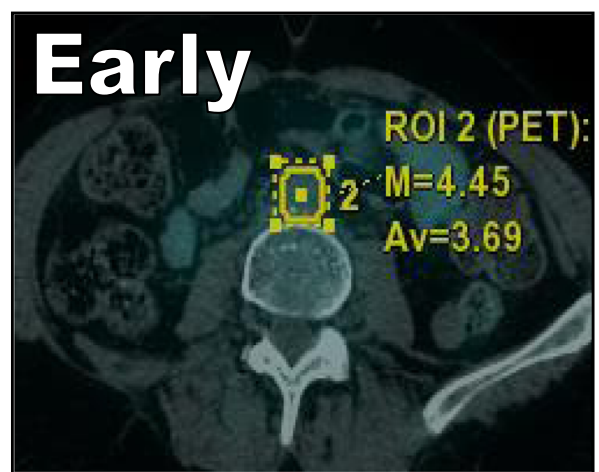

(B)

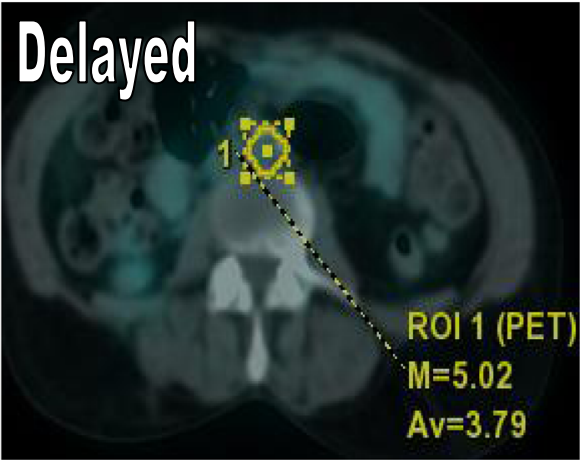

(阿)

Fig. 2 Male patient 58-year-old, non-smoker, non-diabetic, non-hypertensive. He underwent PET/CT for cancer prostate staging. a Non contrast CT images showed dense calcified atheromatous plaque involving 50\% of the abdominal aorta circumference. $\mathbf{b}$ Early PET/CT images after $1 \mathrm{~h}$ showed measurement of SUVmax at the outer wall of the abdominal aorta. c SUVmax measurements at the blood pool (IVC) and liver. d Delayed images after $3 \mathrm{~h}$ showed retention of FDG within the active atheroma with SUVmax higher than the blood pool activity (IVC) and liver denoting grade IV atherosclerotic disease

index percentage was calculated by subtracting the SUVmax in early images from the SUVmax in delayed images and dividing by SUVmax in early images.

\section{( $\frac{\text { SUVmax in delayed images-SUVmax in early images) }}{\text { SUVmax in early images }}$}

The FDG uptake by the aortic arterial wall in the delayed images was graded as follows:

- Grade 1 when arterial FDG activity is equal or less than the blood pool FDG activity.

- Grade 2 when arterial FDG activity is higher than the blood pool FDG activity but less than that of liver.

- Grade 3 when arterial FDG activity is higher than blood pool but equal to the liver FDG activity.
- Grade 4 when arterial FDG activity is higher than blood pool and slightly higher to the liver FDG activity.

\section{$\mathrm{CT}$ findings}

Aortic arterial wall were assessed at four parts of the aorta, including ascending aorta, aortic arch, descending thoracic aorta, and descending abdominal aorta. Within each part intimal HU mean, maximum intimal thickness, degree of intimal changes, degree of aortic calcification, and intra-luminal diameter.

Intimal $\mathrm{HU}$ mean were measured in non-enhanced images by multiple drawing regions of interest including the aortic wall measuring $\mathrm{HU}$ mean in each drawing. Then the mean of all readings were calculated for each part.

The intimal thickness were measured in the contrast enhancing images to differentiate the enhanced luminal blood from the relative less enhancing aortic wall. The degree of intimal changes was shown 

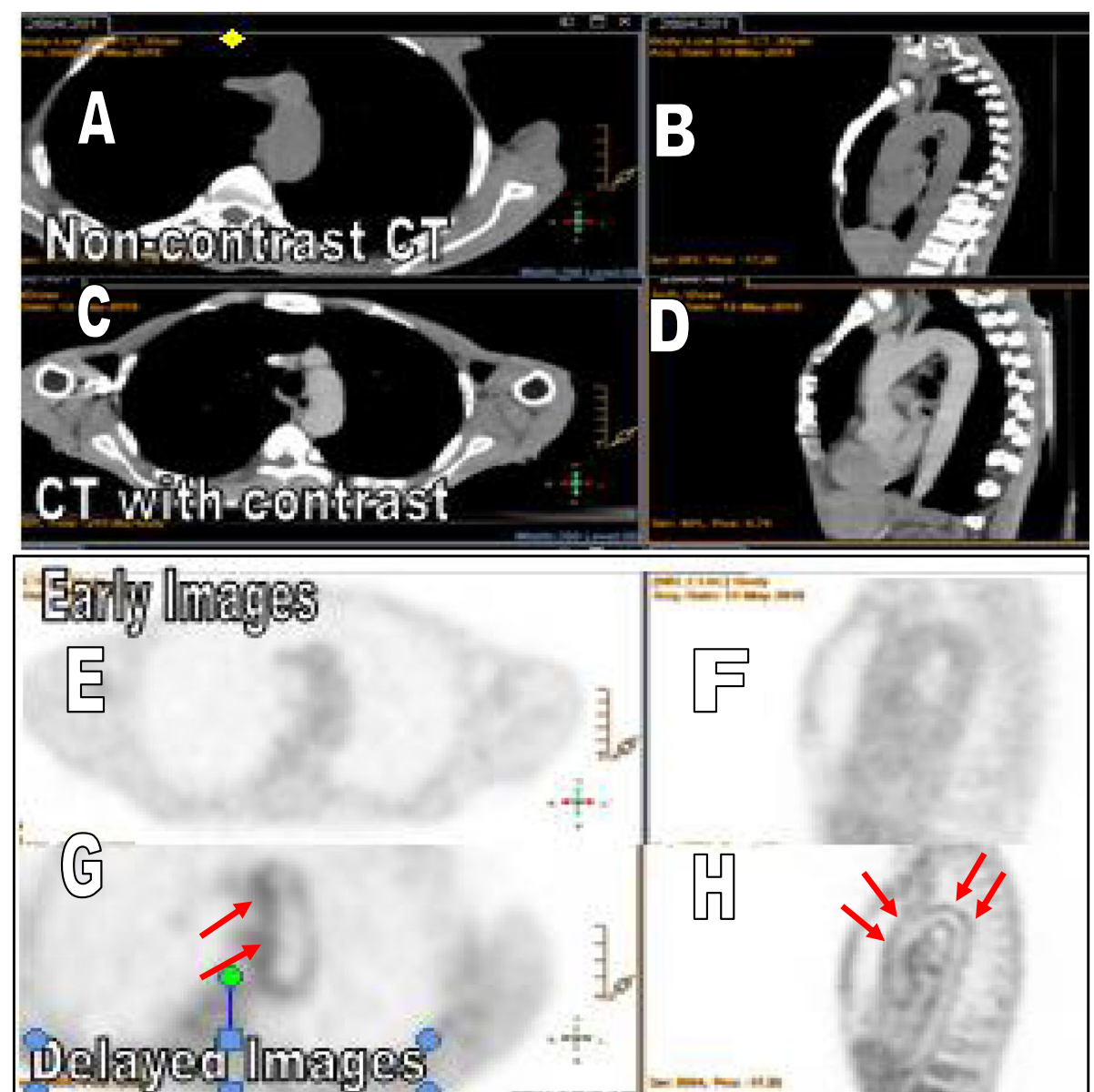

Fig. 3 Male patient 55-year-old, non-smoker, non-diabetic, non-hypertensive. He underwent PET/CT for nasopharyngeal cancer staging. Non contrast $(\mathbf{a}, \mathbf{b})$ and post contrast $\mathrm{CT}$ images $(\mathbf{c}, \mathbf{d})$ showed minimal intimal thickening of the thoracic aorta reaching $3 \mathrm{~mm}$ with no obvious calcification denoting early atherosclerotic disease. Note the difference between the early PET images (e, $\mathbf{f}$ ) and delayed images $(\mathbf{g}, \mathbf{h})$ with retention of FDG within the aortic wall (arrows)

by a percentage of thickenings of the circumference of the aortic wall for each segment. The intimal changes were evaluated qualitatively including outline irregularity with or without calcific changes. Regarding intra-luminal diameter, the internal luminal diameter measured in the largest cross-section of the aortic diameter except in aortic arch where the shortest axis is used. The ratio of the aortic diameter to body surface area was used to correct for differences in individual body build. The degree of aortic calcification was measured in non-enhanced $\mathrm{CT}$ images. It was expressed in the form of percentage of the longitudinal dimensions of each length.

\section{Statistical methods}

Data were statistically described in terms of mean \pm standard deviation $( \pm S D)$, median and range, or frequencies (number of cases) and percentages when appropriate. Stepwise multivariate regression analysis was performed to examine the potential interactions among the entered covariates. The Student $t$ test was used for comparison of paired data; $P$ values less than 0.05 was considered statistically significant. Comparison of numerical variables between the study groups was done using Kruskal-Wallis test with post-hoc multiple 2-group comparisons. Spearman's correlation coefficient $\left(R_{\mathrm{s}}\right)$ was used to measure of the strength of the relationship. $-1 \leq R_{\mathrm{s}} \leq 1$. The absolute value of RS

- $0.00-0.19$ "very weak"

- 0.20-0.39 "weak"

- $0.40-0.59$ "moderate"

- $0.60-0.79$ "strong"

- $0.80-1.0$ "very strong"

While the sign indicate the direction of the correlation either directly proportion (positive) or 
Table $1 \mathrm{CT}$ findings in the 50 patients of study population

\begin{tabular}{|c|c|c|c|c|}
\hline & Ascending aorta & Aortic arch & Descending thoracic aorta & Descending abdominal aorta \\
\hline & Mean \pm SD & Mean \pm SD & Mean \pm SD & Mean \pm SD \\
\hline Luminal diameter & $2.4 \mathrm{~cm} \pm 0.5$ & $2.9 \pm 0.4$ & $2.7 \pm 0.4$ & $2.2 \pm 0.3$ \\
\hline Intimal thickness & $4.4 \mathrm{~mm} \pm 1.8$ & $4.8 \pm 1.75$ & $4.7 \pm 1.6$ & $4.8 \pm 1.7$ \\
\hline Intimal changes & $36.7 \% \pm 26.4$ & $39.2 \pm 27.1$ & $36.7 \pm 29.3$ & $50.5 \pm 36.8$ \\
\hline Intimal HU & $152.2 \pm 139$ & $157.4 \pm 139$ & $347.9 \pm 139$ & $363.3 \pm 360.4$ \\
\hline Wall Calcifications & $9.7 \% \pm 12.5$ & $13.2 \pm 13.8$ & $12.5 \pm 14.8$ & $9.7 \pm 12.5$ \\
\hline
\end{tabular}

inversely proportion (negative). Correlation between various variables was done using Spearman rank correlation equation for non-normal variables/nonlinear monotonic relation. All statistical calculations were done using computer program IBM SPSS (Statistical Package for the Social Science; IBM Corp, Armonk, NY, USA) for Microsoft Windows.

\section{Results}

The study included 50 patients [30 males (60\%) and 20 females $(40 \%)$ ] with male to female mean ratio 1.5 : 1 and their mean age $58.3 \pm 15.7$ years. Eleven patients $(22 \%)$ showed no aortic atherosclerotic changes. Moreover, most of the cases (42\%) had grade III atherosclerotic lesions, whereas grade II and grade IV were detected in $18 \%$ of the cases for each. No patient in our study had grade V. Detailed CT regional aortic findings in our cases were shown in (Table 1). The examined parts of thoracic aorta (ascending, arch and descending parts) as well as the abdominal aorta showed no abnormality regarding their luminal diameter as well as intimal thickness. On the other hand, abdominal descending aorta as well as thoracic descending aorta showed the highest $\mathrm{HU}$ mean $(363.3 \pm 360.4$ and $347.9 \pm 139$ respectively) compared to that of ascending and arch of aorta (152.2 \pm 139 and $157.4 \pm 139$ respectively). Moreover, the highest intimal changes were detected in abdominal aorta $(50.5 \pm 36.8)$ compared to thoracic aorta segments. Wall calcifications were higher in aortic arch and descending aorta $(13.2 \pm 13.8$ and $12.5 \pm 14.8$ respectively) compared to ascending aorta and abdominal aorta that showed similar values. $9.7 \% \pm 12.5$ and $9.7 \pm 12.5$ for each.

Table 2 Dual point blood pool (IVC lumen) SUVs and retention index percentage in the 50 patients of study population

\begin{tabular}{llll}
\hline Blood pool FDG activity & & & \\
\hline & Range & Mean & SD \\
\hline Early SUVmax & 0.9 to 2.2 & 1.492 & 0.3636 \\
Delayed SUVmax & 0.7 to 2.0 & 1.214 & 0.3620 \\
Retention index percentage & -0.4 to $0.2 \%$ & $-0.182 \%$ & 0.1635 \\
\hline
\end{tabular}

Dual point blood pool activity SUVs were done with ROI placed in inferior vena cava (IVC) lumen with calculation of the retention index percentage. Our results denote insignificant higher SUVmax value $1.492 \pm 0.3636$ compared to delayed SUVmax value $1.214 \pm 0.3620$ with retention index percentage $-0.182 \pm 0.1635 \%$ (Table 2). Similarly, hepatic early SUVmax showed insignificant relative higher value $2.82 \pm 0.52$ compared to hepatic late SUVmax $2.2 \pm$ 0.26 with retention index percentage $0.1 \pm 0.03 \%$ (Table 3). On evaluating FDG quantitative parameters in different segments of aorta, all aortic segments (ascending aorta, arch, descending thoracic, and abdominal aorta) showed grade I to II. Moreover, delayed SUVmax showed higher values than the early SUVmax in all segments of the aorta with almost similar values. Regarding retention index percentage of thoracic and abdominal aorta, it showed significantly higher values in comparison to blood pool (IVC) retention index percentage (Tables 4, 5, 6 , and 7).

The relation of FDG activity with CT findings was done within the thoracic and abdominal aorta using test of significance $(P$ value) as well as Spearman's correlation coefficient (Rs value). Regarding PET findings in different segments of the aorta, early SUVmax did not show any significant correlation with $\mathrm{CT}$ findings.

On the other hand, delayed SUVmax of different aortic segments showed significant changes but with no significant correlation with their CT findings $(P<0.05)$ except with calcification.

Table 4 Ascending aorta FDG dual point quantitative parameters in the 50 patients of study population Ascending aorta FDG activity

\begin{tabular}{lllll}
\hline & Range & Mean & SD & $P$ value \\
\hline Early SUVmax & 0.8 to 2.7 & 1.772 & 0.4185 & 0.2 \\
Delayed SUVmax & 0.8 to 3.1 & 2.140 & 0.4901 & 0.02 \\
Retention index percentage & 1 to $50 \%$ & $22 \%$ & 20 & 0.01 \\
Grading of delayed FDG activity & Ranging between grade 1 and 2 \\
\hline
\end{tabular}


Table 3 Dual point hepatic SUVs and Retention index percentage in the 50 patients of study population

\begin{tabular}{llll}
\hline Hepatic FDG activity & & & \\
\hline & Range & Mean & SD \\
\hline Early SUVmax & 1.9 to 3.9 & 2.82 & 0.52 \\
Delayed SUVmax & 1.8 to 2.7 & 2.2 & 0.26 \\
Retention index percentage & -0.1 to $0.3 \%$ & $0.1 \%$ & 0.03 \\
\hline
\end{tabular}

Concerning retention index percentage, their correlations were strong positive in relation to aortic segment intimal changes in descending thoracic aorta and descending abdominal aorta with $R$ s value 0.6 , $0.7,0.6$, and 0.7 respectively. Whereas it was positive with weak to moderate strength in relation to the segments intimal thickness changes in descending thoracic aorta and descending abdominal aorta with $R$ s value $0.3,0.5,0.3$, and 0.4 respectively and similarly to intimal maximum $\mathrm{HU}$ with $R$ s value $0.3,0.4$, 0.4 , and 0.5 (Tables 8 and 9).

\section{Discussion}

This prospective study included 50 patients aiming at quantification of the aortic arterial wall FDG uptake as well as FDG retention index percentage by post 1 and $3 \mathrm{~h}$ post tracer injection, in patient with different risk factors of atherosclerosis. We also correlate the functional PET/CT findings with the abnormal CT findings in those patients using dual point technique.

Most of cases in this study showed CT grade III atherosclerosis (42\%) with absence of grade $\mathrm{V}$ cases. This can be explained by their younger mean age (mean age $55.3 \pm 10.1$ ) with less time allowed for development of atherosclerosis complications. Our study showed that the highest atherosclerotic changes were mainly in the abdominal aorta followed by descending part of descending aorta. This could be attributed to exaggerated and prolonged stress (sheering effect) exerted on abdominal aorta due to turbulent blood flow at the orifices of its branches [7]. On the contrary, aortic calcifications was common in aortic arch; this can attributed to high blood pressure in their wall with

Table 5 Aortic arch FDG dual point quantitative parameters in the 50 patients of study population

\begin{tabular}{lllll}
\hline Aortic arch FDG activity & & & & \\
\hline & Range & Mean & SD & $P$ value \\
\hline Early SUVmax & 1.1 to 2.5 & 1.734 & 0.4074 & 0.3 \\
Delayed SUVmax & 1.1 to 2.9 & 2.050 & 0.4400 & 0.01 \\
Retention index percentage & 0 to 60 & $21 \%$ & 25 & 0.0001 \\
Grading of delayed FDG activity & Ranging between grade 1 and 2 \\
\hline
\end{tabular}

Table 6 Descending thoracic aorta FDG dual point quantitative parameters in the 50 patients of study population

\begin{tabular}{lllll}
\hline Descending thoracic FDG activity & & & & \\
\hline & Range & Mean & SD & $P$ value \\
\hline Early SUVmax & 1.2 to 2.5 & 1.782 & 0.377 & 0.255 \\
Delayed SUVmax & 1.1 to 3.7 & 2.113 & 0.595 & 0.035 \\
$\begin{array}{l}\text { Retention index percentage } \\
\text { Grading of delayed FDG }\end{array}$ & -3 to $65 \%$ & $18 \%$ & 22 & 0.001 \\
activity & Ranging between grade 1 and 2 \\
\hline
\end{tabular}

earlier atherosclerotic changes that healed by fibrosis and calcification [8]. Regarding dual point blood pool activity measured in inferior vena cava lumen, our results showed higher but insignificant SUVmax values in early study compared to late study denoting FDG background activity decreased with time. This is very important including oncological PET that could be delayed as target (lesion) to background ratio will be increased resulting in better detection of the lesion as well as detection of more numbers of lesions. Similar findings were observed on evaluation of hepatic dual point SUVmax. All cases in our study had only PET grade I and II (i.e., less than hepatic activity) arterial wall uptake. This may be also attributed to their relatively young main age with less time to develop complicating atherosclerotic disease [9]. Among pioneered studies evaluating FDG uptake in aortic atherosclerosis was that study conducted by Tatsumi et al. [10]. They concluded that increased aortic wall FDG uptake regions were mostly distinct from the calcification sites. Moreover, FDG uptake is possibly located in areas of metabolic activity of active atherosclerotic changes. Meirelles et al. [11] also studied 18F-FDG uptake as a tool detecting metabolically active atherosclerotic plaque. They detailed the frequency and stability of 18F-FDG uptake and vascular calcifications in the thoracic aorta on serial 18F-FDG PET/ CT studies and correlated the findings with clinical data and risk factors for cardiovascular diseases. They concluded that calcifications were stable over time, but 18F-FDG uptake changed in more than half of the patients postulating that inflammation in

Table 7 Descending abdominal aorta FDG dual point quantitative parameters in the 50 patients of study population

\begin{tabular}{lllll}
\hline Abdominal aorta FDG activity & & & & \\
\hline & Range & Mean & SD & $P$ value \\
\hline Early SUVmax & 1.0 to 2.9 & 1.954 & 0.433 & 0.555 \\
Delayed SUVmax & 1.2 to 3.4 & 2.226 & 0.546 & 0.0213 \\
Retention index percentage & 0 to $45 \%$ & $15 \%$ & 17 & 0.0001 \\
Grading of delayed FDG activity & Ranging between grade 1 and 2 \\
\hline
\end{tabular}


Table 8 Correlation between FDG findings and CT findings in the 50 patients of study population within the descending thoracic aorta

Descending Thoracic Aorta

\begin{tabular}{|c|c|c|c|c|c|c|c|c|c|c|}
\hline & \multicolumn{2}{|c|}{ Intimal changes $\%$} & \multicolumn{2}{|c|}{ Luminal diameter } & \multicolumn{2}{|c|}{ Intimal thickness } & \multicolumn{2}{|c|}{ Intimal HU } & \multicolumn{2}{|c|}{ Calcifications \% } \\
\hline & $P$ value & Rs value & $P$ value & Rs value & $P$ value & Rs value & $P$ value & Rs value & $P$ value & Rs value \\
\hline Early SUVmax & 0.777 & 0.04 & 0.722 & 0.05 & 0.140 & 0.2 & 0.292 & 0.1 & 0.681 & 0.06 \\
\hline Delayed SUVmax & 0.01 & 0.21 & 0.043 & 0.2 & 0.052 & 0.2 & 0.006 & 0.06 & 0.006 & 0.2 \\
\hline Retention index \% & 0.000 & 0.6 & 0.022 & 0.03 & 0.046 & 0.3 & 0.060 & 0.4 & 0.002 & 0.04 \\
\hline
\end{tabular}

atheroma can progress or regress in a waxing and waning process. In the current study, these findings were quite congruent with our correlations results. We denoted that the correlation between arterial wall FDG uptake in the early and delayed studies to the percentage of aortic calcification were insignificant $(P>0.2)$ as well as the retention index percentage of arterial wall FDG activity showed no correlation (Rs near zero). Tawakol et al. [12] were the first to link plaque inflammation regarding histological macrophages content to 18F-FDG uptake in plaques of 17 patients scheduled for carotid endarterectomy. They found a significant correlation between the PET signal from the carotid plaques and the macrophage staining from the corresponding histological sections with $(r=0.70$ and $P<0.0001)$.

In a prospective study by Rudd et al. [13], they studied the relationships of the 18F FDG uptake to multi-variables in risk factors including calcification and regional arterial inflammation. The study included 41 patients who underwent vascular FDG PET/CT imaging who had either vascular disease or other multiple risk factors. They found strong associations between FDG uptake in neighboring arteries (right versus left carotid, $r=0.91, P<0.001$; aortic arch versus ascending aorta, $r=0.88, P<0.001$ ). They concluded that plaque inflammation and calcification rarely overlap, supporting the theory that calcification represents a late stage of atherosclerosis. Inflammation in one arterial territory is associated with inflammation elsewhere. They suggested that FDG PET imaging could be used as an important marker of both atherosclerotic disease activity and drug effectiveness. Many studies are now prospectively done to determine the role of this technique in clinical risk prediction. In the current study, instead of histology-based approaches and biochemical profile, we detect CT morphological changes that indicate or suggest either early developing or complicated atherosclerosis in aortic arterial wall at the four parts of the aorta, including intimal HU mean value, maximum intimal thickness, degree of intimal changes, degree of aortic calcification, and intra-luminal diameter. Moreover, our early and delayed SUVmax of different segments of aorta showed insignificant difference. This is expected as atherosclerosis is a systemic disease involving the whole cardiovascular endothelium. Different segments only differ in their atherosclerotic grades and/or stages of healing.

\section{Conclusion}

* Aortic wall FDG uptake can be used as an additional parameter as well as a biomarker on evaluation of the arterial atherosclerotic activity.

- Delayed post $3 \mathrm{~h}$ FDG imaging is more accurate than the routine early post $1 \mathrm{~h}$ imaging in evaluating the atherosclerotic activity.

* Retention FDG index is positively correlated with arterial CT changes including arterial intimal thickness, percentage of intimal changes, and $\mathrm{HU}$ of the arterial wall.

* FDG uptake may be used as a diagnostic index for atheroma cellular content and hence vulnerability.

Table 9 Correlation between FDG findings and CT findings in the 50 patients of study population within the descending abdominal aorta

\begin{tabular}{|c|c|c|c|c|c|c|c|c|c|c|}
\hline \multicolumn{11}{|c|}{ Descending abdominal aorta } \\
\hline & \multicolumn{2}{|c|}{ Intimal changes \% } & \multicolumn{2}{|c|}{ Luminal diameter } & \multicolumn{2}{|c|}{ Intimal thickness } & \multicolumn{2}{|c|}{ Intimal HU } & \multicolumn{2}{|c|}{ Calcifications\% } \\
\hline & $P$ value & Rs value & $P$ value & Rs value & $P$ value & Rs value & $P$ value & Rs value & $P$ value & Rs value \\
\hline Early SUVmax & 0.780 & 0.04 & 0.0 & 0.5 & 0.456 & 0.1 & 0.817 & 0.03 & 0.102 & -0.2 \\
\hline Delayed SUVmax & 0.120 & 0.04 & 0.123 & 00.5 & 0.01 & 0.2 & 0.0067 & 0.3 & 0.003 & -0.1 \\
\hline Retention index \% & 0.011 & 0.7 & 0.02 & 0.02 & 0.023 & 0.4 & 0.017 & 0.5 & 0.475 & 0.0 \\
\hline
\end{tabular}




\section{Abbreviations}

CT: Computed tomography; FDG: Fluorodeoxyglucose; IVC: Inferior vena cava; PET: Positron emission tomography; ROls: Regions of interest; Rs: Spearman's correlation coefficient; SD: Standard deviation; SUV: Standard uptake value; SUVmax: Maximum SUV

\section{Acknowledgments}

Not applicable.

\section{Authors' contributions}

$\mathrm{AAH}$ put the idea of the study. Editor of the manuscript. Participated in the study design. NDE participated in the study design, patients collection, clinical assessment, and performed the statistical analysis. Both authors read and approved the final manuscript.

\section{Funding}

Not applicable (no funding received for this study).

\section{Availability of data and materials}

All the datasets used and analyzed in this study are available with the corresponding author on reasonable request.

\section{Ethics approval and consent to participate}

Written informed consent was signed by all patients before the examination. The study was approved by the research committee of faculty of medicine, kasr Alainy hospital. Cairo University 2018. No reference number provided as the committee just say yes or no according to the system in our faculty of medicine at 2018 (date of starting of this research).

\section{Consent for publication}

All patients included in this research were fully conscious and older than 16 years old and gave written informed consent to publish the data contained within this study.

\section{Competing interests}

The authors declare that they have no competing interests.

\section{Author details}

${ }^{1}$ Radiodiagnosis, Faculty of Medicine, Cairo University, Giza, Egypt. ${ }^{2}$ Nuclear Medicine, Department of oncology and nuclear medicine, Faculty of Medicine, Cairo University, Giza, Egypt.

Received: 25 August 2019 Accepted: 9 January 2020

Published online: 14 January 2020

\section{References}

1. Montecucco F, Mach F. Atherosclerosis is an inflammatory disease. Seminars in immunopathology; 2009: Springer. https://link.springer.com/article/10.1 007/s00281-009-0146-7.

2. Ferrari E, Vidal R, Chevallier T et al (1999) Atherosclerosis of the thoracic aorta and aortic debris as a marker of poor prognosis: benefit of oral anticoagulants. J Am Coll Cardiol 33(5):1317-1322

3. Tarkin JM, Dweck MR, Evans NR et al (2016) Imaging atherosclerosis. Circ Res 118(4):750-769

4. Cavalcanti Filho JLG, RdSL L, LdSM N et al (2011) PET/CT and vascular disease: current concepts. Eur J Radiol 80(1):60-67

5. Virmani R, Kolodgie FD, Burke AP et al (2005) Atherosclerotic plaque progression and vulnerability to rupture: angiogenesis as a source of intraplaque hemorrhage. Arterioscler Thromb Vasc Biol 25(10):2054-2061

6. Cybulsky MI, liyama K, Li H et al (2001) A major role for VCAM-1, but not ICAM-1, in early atherosclerosis. J Clin Invest 107(10):1255-1262

7. Beşer CG, Karcaaltıncaba M, Çelik HH et al (2016) The prevalence and distribution of the atherosclerotic plaques in the abdominal aorta and its branches. Folia Morphol (Warsz) 75(3):364-375

8. Fleg JL, Stone GW, Fayad ZA, Granada JF, Hatsukami TS, Kolodgie FD, Ohayon J, Pettigrew R, Sabatine MS, Tearney GJ, Waxman S (2012) Detection of high-risk atherosclerotic plaque: report of the NHLBI Working Group on current status and future directions. JACC Cardiovasc Imaging 5(9):941-955

9. Shammas NW (2007) Epidemiology, classification, and modifiable risk factors of peripheral arterial disease. Vasc Health Risk Manag 3(2):229
10. Tatsumi M, Cohade C, Nakamoto Y et al (2003) Fluorodeoxyglucose uptake in the aortic wall at PET/CT: possible finding for active atherosclerosis. Radiology 229(3):831-837

11. Meirelles GSP, Gonen M, Strauss HW (2011) 18: frequency and stability on serial Scansf-fdg uptake and calcifications in the thoracic aorta on positron emission tomography/computed tomography examinations: frequency and stability on serial scans. J Thorac Imaging 26(1):54-62

12. Rudd JH, Myers KS, Bansilal S et al (2009) Relationships among regional arterial inflammation, calcification, risk factors, and biomarkers: a prospective fluorodeoxyglucose positron-emission tomography/computed tomography imaging study. Circulation 2(2):107-115

13. Tawakol A, Migrino RQ, Bashian GG et al (2006) In vivo 18Ffluorodeoxyglucose positron emission tomography imaging provides a noninvasive measure of carotid plaque inflammation in patients. J Am Coll Cardiol 48:1818-1824

\section{Publisher's Note}

Springer Nature remains neutral with regard to jurisdictional claims in published maps and institutional affiliations.

\section{Submit your manuscript to a SpringerOpen ${ }^{\circ}$ journal and benefit from:}

- Convenient online submission

- Rigorous peer review

- Open access: articles freely available online

High visibility within the field

- Retaining the copyright to your article

Submit your next manuscript at $\boldsymbol{\nabla}$ springeropen.com 